\title{
Correction to: the disruption of mitochondrial axonal transport is an early event in neuroinflammation
}

Oihana Errea ${ }^{1}$, Beatriz Moreno ${ }^{1}$, Alba Gonzalez-Franquesa ${ }^{2}$, Pablo M. Garcia-Roves ${ }^{2,3,4}$ and Pablo Villoslada ${ }^{1,5^{*}}$

\section{Correction}

After publication of the article [1], it has been brought to our attention that the full funding acknowledgement is missing from the original article. It should also include the following -

"This work was supported by the Instituto de Salud Carlos III with FEDER funds (Otra forma de hacer Europa) from the European Commission (FIS: PI12/ 01823)".

\section{Author details}

'Center of Neuroimmunology, Institut d'Investigacions Biomèdiques August Pi Sunyer (IDIBAPS), Cellex Building, Laboratory 3A, Casanova 145, 08036 Barcelona, Spain. ${ }^{2}$ Diabetes and Obesity Research Laboratory, Institut d'Investigacions Biomèdiques August Pi Sunyer (IDIBAPS), 08036 Barcelona, Spain. ${ }^{3}$ Spanish Biomedical Research Center in Diabetes and associated disorders (CIBERDEM), University of Barcelona, 08907 Barcelona, Spain. ${ }^{4}$ Department of Physiological Sciences II, University of Barcelona, 08907 Barcelona, Spain. ${ }^{5}$ University of California, San Francisco, USA.

Published online: 23 October 2017

\section{Reference}

1. Errea O, Moreno B, Gonzalez-Franquesa A, Garcia-Roves P, Villoslada P. The disruption of mitochondrial axonal transport is an early event in neuroinflammation. J Neuroinflammation. 2015;12(1):152. doi:10.1186/ s12974-015-0375-8.

\footnotetext{
* Correspondence: pvillosl@clinic.cat

${ }^{1}$ Center of Neuroimmunology, Institut d'Investigacions Biomèdiques August Pi Sunyer (IDIBAPS), Cellex Building, Laboratory 3A, Casanova 145, 08036 Barcelona, Spain

${ }^{5}$ University of California, San Francisco, USA
} 